\title{
The Advantage of an Endoscopic Submucosal Tunneling Technique for Rectal Carcinoid Tumors
}

\author{
Hideki Kobara, Hirohito Mori, Li Chei, Shintaro Fujihara, Noriko Nishiyama, and Tsutomu Masaki \\ Department of Gastroenterology and Neurology, Kagawa University Faculty of Medicine, Kagawa, Japan
}

Endoscopic treatment can be a curative option for small carcinoid tumors with an extremely low risk of metastasis. Since most carcinoid tumors are characterized by a specific growth pattern in the submucosal (SM) layer, specialized endoscopic techniques for deeper resection to achieve clear vertical margins are needed. The endoscopic submucosal dissection (ESD) method in the SM space is superior to conventional endoscopic mucosal resection. However, the standard ESD technique sometimes fails to provide complete deep SM dissection due to insufficient SM lifting. Here, to resolve this problem, we describe our initial experience with an endoscopic SM tunneling technique that is effective for treating rectal carcinoid tumors. (Gut Liver 2017;11:735737)

Key Words: Carcinoid tumor; Endoscopy, gastrointestinal

\section{INTRODUCTION}

Traditionally, surgical treatment was performed for carcinoid tumors. Recently, minimally invasive endoscopic treatment can be curative for small carcinoid tumors $(<10 \mathrm{~mm})$ with an extremely low risk of metastasis. ${ }^{1}$ Endoscopic treatment for carcinoid tumors requires special techniques for deeper resection to achieve clear vertical margins, since most of the tumors extend into the submucosal (SM) layer. ${ }^{2}$ In the point of view, endoscopic submucosal dissection (ESD) method takes several advantages of enabling secure submucosal dissection and precise histological assessment of the resected specimen over conventional endoscopic mucosal resection (EMR). ${ }^{3}$ However, standard ESD technique sometimes fails in completion of deep submucosal dissection due to insufficient submucosal lifting. After the whole circumferential incision, submucosal injection tends to dissipate easily, and sometimes it becomes difficult and hazardous to work in the SM space. To resolve this issue, we here describe initial experience of endoscopic submucosal tunneling technique effective for rectal carcinoid tumor.

\section{CASE REPORT}

A 45-year-old woman presented with a definite carcinoid tumor ( $3 \mathrm{~mm}$ in diameter) located in the lower of rectum. EUS revealed the tumor originating in the SM layer, without infiltration of the muscularis propria (MP) layer. While obtaining informed consent according to the consent forms approved by the Institutional Review Board of Kagawa University Hospital (IRB number: H25-030), the following strategy with retroflexed endoscopic view was designed. First, after circumferential marking with a margin of about $10 \mathrm{~mm}$, a 10-mm entry toward the

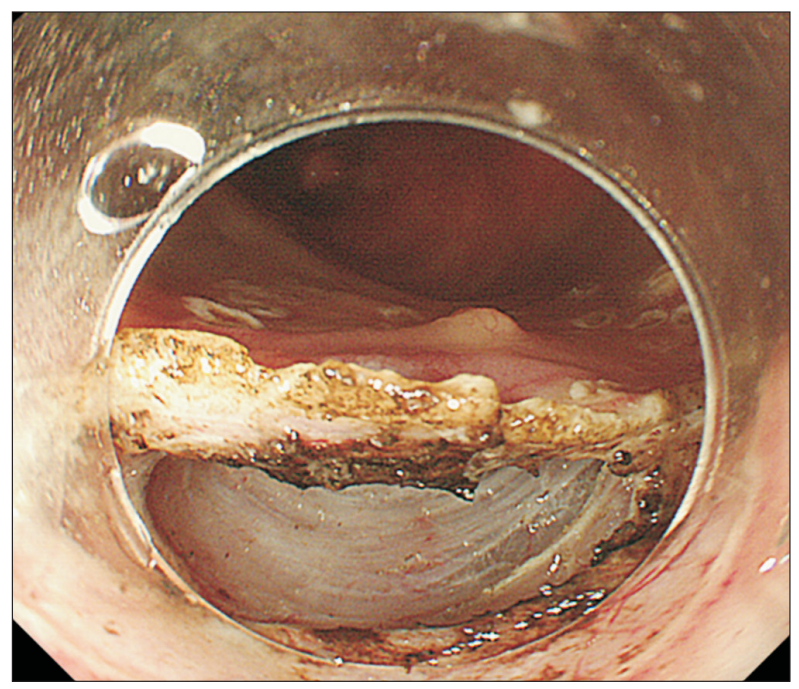

Fig. 1. Endoscopic view showing the creation of a submucosal tunnel with a $10-\mathrm{mm}$ entry.

Correspondence to: Hideki Kobara

Department of Gastroenterology and Neurology, Kagawa University Faculty of Medicine, 1750-1 Ikenobe, Miki, Kita, Kagawa 761-0793, Japan

Tel: +81-87-891-2156, Fax: +81-87-891-2158, E-mail: kobara@med.kagawa-u.ac.jp

Received on December 1, 2016. Revised on March 22, 2017. Accepted on April 20, 2017.

pISSN 1976-2283 eISSN 2005-1212 https://doi.org/10.5009/gnl16580

@ This is an Open Access article distributed under the terms of the Creative Commons Attribution Non-Commercial License (http://creativecommons.org/licenses/by-nc/4.0) which permits unrestricted non-commercial use, distribution, and reproduction in any medium, provided the original work is properly cited. 


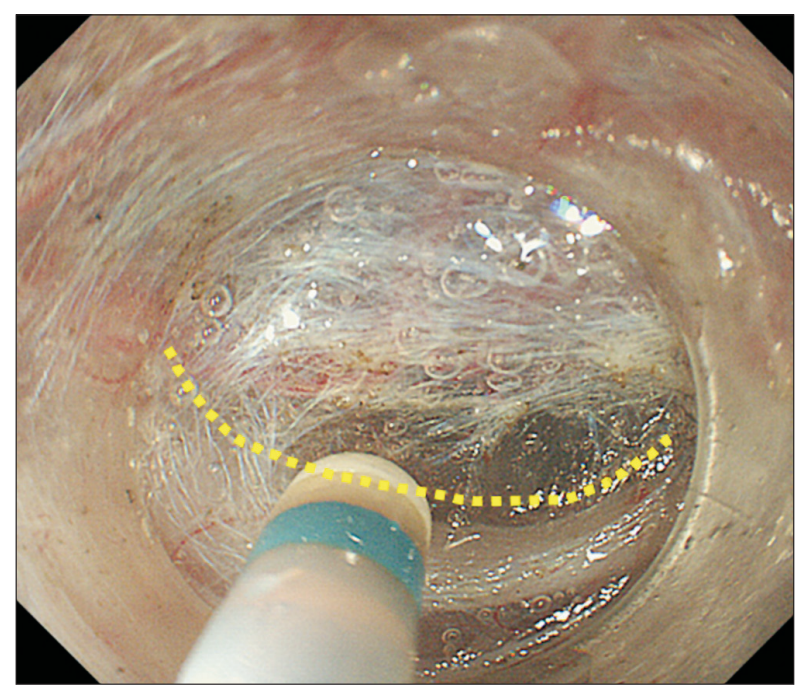

Fig. 2. Endoscopic findings showing a cut line (yellow dotted line) that is visually identified between the deep submucosal (SM) and muscularis propria layers; the SM tunnel exhibits sufficient SM lifting, as indicated by an injected solution.

submucosa (Fig. 1) was made using a needle knife (KD 650Q; Olympus, Tokyo, Japan) and a longer transparent cap (Elastic Touch F-030; Top Corp., Tokyo, Japan) on the purpose of easy insertion of the endoscope into the deep SM layer. Next, while creating submucosal tunnel, a sufficient submucosal lifting with several milliliters of $0.4 \%$ hyaluronate sodium solution (MucoUp; Johnson \& Johnson K.K., Tokyo, Japan) appeared through the tunnel, demonstrating clearly the cut line between deep SM and MP layer (Fig. 2). Finally, after the cut line was dissected toward the opposite site, standard ESD that involves the whole circumferential cutting and additional dissection was completed without associated complications. The procedure time was 39 minutes. All procedures were performed by a single endoscopist (H.K.) who has successfully experienced more than 300 ESD cases for gastrointestinal (GI) neoplasms. Histological examination revealed en bloc resection of a neuroendocrine tumor (NET) grade 1 that showed negative lateral and vertical margin, Ki-67 index $\leq 2 \%$, and mitotic counts $<2 / 10$ high-power field. Three months later, the follow-up examinations with sigmoidoscopy and computed tomography revealed no recurrences of the tumor. The patient had an excellent outcome with minimal invasive treatment.

\section{DISCUSSION}

Currently, endoscopic submucosal tunneling technique, collectively best termed as submucosal endoscopy, is a promising procedure for diagnosis and therapeutic intervention possible in this submucosal space. ${ }^{4-6}$ The efficacy of this technique for superficial esophageal squamous cell neoplasms, as well as other methods, like popular peroral endoscopic myotomy (POEM), ${ }^{7}$ submucosal tunnel endoscopic resection (STER) ${ }^{8}$ for subepithelial tumors originating in the MP layer, have currently been described as a new treatment strategy on esophageal ESD. ${ }^{9}$ The major procedure of the tunneling method is to set first the distal demarcation, then make a $10-\mathrm{mm}$ entry at proximal site followed by creating a submucosal tunnel by SM dissection. During the procedure, the usage of a short cap is essential to facilitate the navigation through the SM space. The biggest advantage of this technique is the capability of dissecting the deep SM layer owing to sufficient submucosal lifting by creating a tunnel.

Among various endoscopic procedures, conventional EMR, modified EMR using other assistant devices such as a ligation band or a suction cap, and ESD have been generally introduced for endoscopic resection of small rectal carcinoid tumors. A systematic review with a meta-analysis of 10 studies with 650 cases demonstrated that complete resection rates were significantly higher in the ESD and modified EMR (m-EMR) groups compared with the conventional EMR group, and also m-EMR appeared to be comparable with ESD. ${ }^{10}$ However, several issues remain in each method. The depth of SM layer resected by the $\mathrm{m}$-EMR depends on the discretion of the operator, thus having the possibility of inaccurate resection and burning effects of vertical margin. This technical issue may make the accurate histological assessment difficult. Whereas, ESD that has the advantage of deep SM layer dissection under direct vision may overcome this issue, providing the high rate of complete resection. Instead, standard ESD needs sufficient SM lifting to accurately dissect the deep SM layer. Thus, the application of submucosal tunneling method can strengthen the ESD methodology.

This method has several limitations of technical aspects and time efficacy. The first limitation is the requirement for an experienced endoscopist to be familiar with ESD techniques. A risk factor associated with the perforation rate may depend on the level of dissected layer such as superficial SM or deep SM or MP layer with the tunneling method. Notably, STER for subepithelial tumors and POEM in which the dissection of the MP layer is needed may be remarked as an advanced technology with unavoidable risk of the perforation during procedures. In contrast, the tunneling method for early esophageal tumors showed no differences of the complication rate compared with standard $\mathrm{ESD},{ }^{9}$ revealing that the method is acceptable for dissecting SM layer safely. With regard to the resection of carcinoid tumor characterized by specific growth pattern in the SM layer, high technical skills are required to dissect the submucosa immediately above the MP, recognized as the most technically difficult part of all procedural steps, which is crucial for complete resection with negative vertical margin. Thus, the perforation rate of the technique may be higher than that of ESD indicated for superficial GI neoplasms. In this situation, the usage of a safe device such as grasping type of scissors forceps (Clutch Cutter; Fujifilm, Tokyo, Japan) may overcome the issue of increased 
perforation risk and be acceptable for any levels of ESD performers, compared with that of needle knives. Regarding time efficacy, the meta-analysis revealed that the comparison of the procedure time between the m-EMR group and the ESD group did not show statistically significant differences. ${ }^{10}$ However, the procedure time of the method described in our case seems to be relatively longer compared with other methods. Prospective studies with large samples are needed to further investigate time efficacy and technical advantage of this method including the learning curve.

In conclusion, ESD procedure involving submucosal tunneling technique may be a reasonable option for histologically en bloc resection of small carcinoid tumors, characterized by specific growth pattern in the SM layer.

\section{CONFLICTS OF INTEREST}

No potential conflict of interest relevant to this article was reported.

\section{REFERENCES}

1. Park HW, Byeon JS, Park YS, et al. Endoscopic submucosal dissection for treatment of rectal carcinoid tumors. Gastrointest Endosc 2010;72:143-149.

2. Matsumoto T, lida M, Suekane H, Tominaga M, Yao T, Fujishima M. Endoscopic ultrasonography in rectal carcinoid tumors: contribu- tion to selection of therapy. Gastrointest Endosc 1991;37:539-542.

3. Zhou PH, Yao LQ, Qin XY, et al. Advantages of endoscopic submucosal dissection with needle-knife over endoscopic mucosal resection for small rectal carcinoid tumors: a retrospective study. Surg Endosc 2010;24:2607-2612.

4. Sumiyama K, Gostout CJ, Rajan E, Bakken TA, Knipschield MA, Marler RJ. Submucosal endoscopy with mucosal flap safety valve. Gastrointest Endosc 2007;65:688-694.

5. Kobara H, Mori H, Rafiq K, et al. Submucosal tunneling techniques: current perspectives. Clin Exp Gastroenterol 2014;7:67-74.

6. Kobara H, Mori H, Fujihara S, et al. Bloc biopsy by using submucosal endoscopy with a mucosal flap method for gastric subepithelial tumor tissue sampling (with video). Gastrointest Endosc 2013;77:141-145.

7. Inoue $\mathrm{H}$, Minami H, Kobayashi $\mathrm{Y}$, et al. Peroral endoscopic myotomy (POEM) for esophageal achalasia. Endoscopy 2010;42:265271

8. Liu BR, Song JT. Submucosal tunneling endoscopic resection (STER) and other novel applications of submucosal tunneling in humans. Gastrointest Endosc Clin N Am 2016;26:271-282.

9. Arantes V, Albuquerque W, Freitas Dias CA, Demas Alvares Cabral MM, Yamamoto H. Standardized endoscopic submucosal tunnel dissection for management of early esophageal tumors (with video). Gastrointest Endosc 2013;78:946-952.

10. Zhou X, Xie H, Xie L, Li J, Cao W, Fu W. Endoscopic resection therapies for rectal neuroendocrine tumors: a systematic review and meta-analysis. J Gastroenterol Hepatol 2014;29:259-268. 